This is a postprint version of the following published document:

Valverde-Albacete, F. J., Peláez-Moreno, C. \& Campo, C. del (2015). Activating Generalized Fuzzy Implications from Galois Connections. In Magdalena, L., Verdegay, J. L. \& Esteva, F. (eds.). Enric Trillas: A Passion for Fuzzy Sets A Collection of Recent Works on Fuzzy Logic. (pp. 201-212). (Studies in Fuzziness and Soft Computing; 322). Springer International Publishing

DOI: http://dx.doi.org/10.1007/978-3-319-16235-5_15

(C) 2015 Springer International Publishing Switzerland 


\title{
Activating Generalized Fuzzy Implications from Galois Connections
}

\author{
Francisco J. Valverde-Albacete ${ }^{1 *}$, Carmen Peláez-Moreno ${ }^{2 \dagger}$ \\ and Cristina del Campo ${ }^{3}$ \\ ${ }^{1}$ Depto. Lenguajes y Sistemas Informáticos, UNED, Madrid, Spain \\ e-mail: fva@1si.uned.es \\ ${ }^{2}$ Depto. Teoría de Señal y Comunicaciones, UC3M, Madrid, Spain \\ e-mail: carmen@tsc.uc3m.es \\ ${ }^{3}$ Depto. Estadística e Investigación Operativa II, UCM, Madrid, Spain \\ e-mail: campocc@ccee.ucm.es
}

\begin{abstract}
This paper deals with the relation between fuzzy implications and Galois connections, trying to raise the awareness that the fuzzy implications are indispensable to generalise Formal Concept Analysis. The concrete goal of the paper is to make evident that Galois connections, which are at the heart of some of the generalizations of Formal Concept Analysis, can be interpreted as fuzzy incidents. Thus knowledge processing, discovery, exploration and visualization as well as data mining are new research areas for fuzzy implications as they are areas where Formal Concept Analysis has a niche.
\end{abstract}

Keywords Semiring theory; Semiring-valued extensions of FCA; Fuzzy implications; Residuation; Philosophical foundations

\section{Introduction}

Our knowledge of complex systems is usually incomplete and even in the cases when it is complete, it is difficult to use. Therefore, in both situations, we have to trust in expert's statements. Fuzzy Logic is a methodology to model those statements that usually belong to natural language instead of being made in mathematical language.

\footnotetext{
${ }^{*}$ F.J. Valverde-Albacete—was partially supported by EU FP7 project LiMoSINe, (contract 288024).

†C. Peláez-Moreno-was partially supported by the Spanish Government-CICYT project 2011-268007/TEC.
} 
In particular, fuzzy implications were introduced and studied in the literature as a generalization of classical implications to represent experts knowledge of the type "If..., then..." and to perform inferences in any rule-based system. In the ample bibliography on the subject (see for example [1-4]) different approaches to the concept of fuzzy implication can be found. Yet, an implication on a subset $E$ is usually understood as an application $\mathscr{J}$ on the fuzzy set $F(E)=[0,1]^{E}$ as

$$
\begin{gathered}
\mathscr{J}:[0,1]^{E} \times[0,1]^{E} \rightarrow[0,1]^{E \times E} \\
(\mu, \sigma) \mapsto \mu \rightarrow \sigma
\end{gathered}
$$

However, when such an implication $\mu \rightarrow \sigma$ can be functionally expressed, it is represented through a two variable function $(\mu \rightarrow \sigma)(x, y)=J(\mu(x), \sigma(y))$ for every $(x, y) \in E \times E$, where $J$ is a numerical function defined on the square unit $J:[0,1] \times[0,1] \longrightarrow[0,1]$. This function is called an implication operator because it generates a fuzzy implication. Most of the implication functions used in fuzzy logic are built from De Morgan triplets based on the formalism of Boolean logic, intuitionistic logic or quantum logic. See for example the seminal works [5-7] or the recent and very complete overview on fuzzy implication functions [8].

Implications are crucial to generalize the basic framework of Formal Concept Analysis (FCA) [9, 10]. Recall that this is a theory of concrete lattices arising from certain Galois connections [11] between two sets induced by a binary incidence relation. It finds concrete applications in data mining, exploratory analysis, knowledge management and information retrieval, among others [12-14].

Perhaps the earliest and more developed generalization is that of Formal Concept Analysis in a Fuzzy Setting (FCAf), where incidences are allowed to have values in a fuzzy algebra $L$ which is also a complete lattice $[15,16]$. Note that such fuzzy algebras can alternatively be described as fuzzy semirings [17]. An independently motivated generalisation of FCA, $\mathscr{K}$-Formal Concept Analysis (KFCA), uses an idempotent semifield $\mathscr{K}$-a kind of semiring with a multiplicative group structureas the range of the relation [18]. Whereas fuzzy semirings are mostly used to capture a "degree of truth", semifields are used to capture the concept of "cost" or, dually, "utility".

It has already been determined that the condition for an algebra to induce a flavour of FCA is that it be a complete residuated lattice [16]. Indeed, the presence of a residuated implication is a prerequisite to define the polars of the Galois connection. On the other hand, FCA has universal representation capabilities for complete lattices, which extends to the representation of (boolean) implications between attributes [10, Sect. 2.3], and this has been extended to FCAf [19], but not to idempotent semifields.

It is intriguing that these algebras induce Galois Connections and FCA inasmuch as idempotent semifields are as different as a naturally-ordered semiring can be from prototypical fuzzy semirings_-like $\langle[0,1]$, max, $\min , 0,1\rangle$-in a sense made evident in this paper. In fact, idempotent semifields do not fulfill some of the more restrictive or technical conditions for an algebra $L$ to define an $L$-fuzzy set [20]: in particular, in an idempotent semifield the identity is never an infinity element. However, already 
seminal work on fuzzy sets considered the possibility that $[0,1]$ in (1) be a partially ordered set [21, footnote 3].

Unsurprisingly, one of the notoriously overlooked abstractions of fuzzy semirings and idempotent semifields are dioids, or naturally-ordered semirings whose zero is

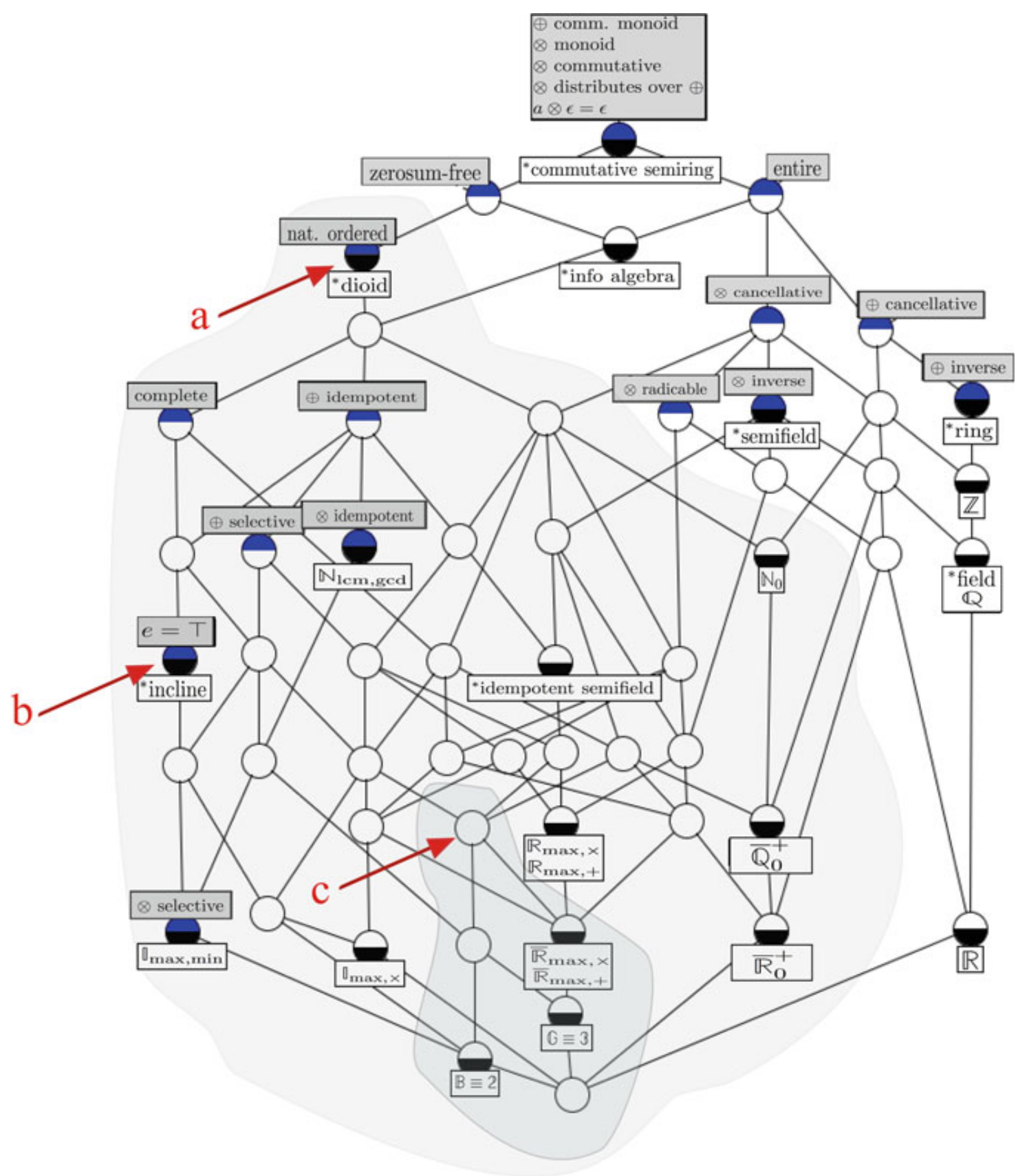

Fig. 1 Concept lattice of dioids in the context of commutative semirings. Concept lattice of a choice of abstract (leading asterisk, white label) and concrete (white label) commutative semirings and their properties (gray label) mentioned in the text. Each node is a concept of abstract algebra: its properties are obtained from the gray labels in nodes upwards, and its structures from the white labels in nodes downwards. The picture is related to the chosen sets of properties and algebras and does not fully reflect the structure of the class of semirings. We have chosen to highlight dioids (a), and, within them, inclines (b) and complete idempotent semifields (c) 
the bottom in the order. The naturalness of the order comes from its compatibility with the multiplication,

$$
\forall a, b, c \in S, a \leq b \Longleftrightarrow c \otimes a \leq c \otimes b
$$

Dioids are already residuated so complete dioids are already complete residuated lattices (see Fig. 1), hence FCA-inducing. Furthermore, semiring $\mathbb{B}$ is embedded in both fuzzy semirings and idempotent semifields. Note that [22] already asked for a revisiting of idempotent semifields and the investigation of their relationship to fuzzy algebras.

In this paper we argue that in future research regarding fuzzy implications the existence of such Galois connections and generalized forms of FCA should be among the first and foremost applications.

For that purpose, we revisit some properties of dioids and their semimodules in Sect. 2, highlighting the formal similarities of the definitions of Galois connections between them. We then present in Sect. 3 some applications of generalized formal concept analysis to emphasize its importance as an application field and last we offer some conclusions.

\section{Preliminaries: Semirings and Semimodules}

To make this work self-contained, we introduce basic notations used in the text and we briefly mention some of the results employed in the rest of the paper. See [17] for a comprehensive introduction to this subject.

Recall that a semiring is an algebra $\mathscr{S}=\langle S, \oplus, \otimes, \varepsilon, e\rangle$ whose additive structure, $\langle S, \oplus, \varepsilon\rangle$, is a commutative monoid and whose multiplicative structure, $\langle S \backslash\{\varepsilon\}, \otimes, e\rangle$, is a monoid with multiplication distributing over addition from right and left and with additive neutral element absorbing for $\otimes$, i.e. $\forall a \in S, \varepsilon \otimes a=\varepsilon$ [17]. A semiring $\mathscr{S}$ is:

1. zerosumfree iff $a \oplus b=\varepsilon \Rightarrow a=\varepsilon$ and $b=\varepsilon$.

2. entire iff $a \otimes b=\varepsilon \Rightarrow a=\varepsilon$ or $b=\varepsilon$.

3. positive or an information algebra iff it is both zerosumfree and entire.

4. partially-ordered iff there is an $\operatorname{order}\langle S, \preccurlyeq\rangle$ compatible with addition and multiplication, such that for all $a, b, c \in S$, if $a \preccurlyeq b$ then $a \oplus c \preccurlyeq b \oplus c, a \otimes c \preccurlyeq b \otimes c$ and $c \otimes a \preccurlyeq c \otimes b$.

Note that, in partially-ordered semirings, if $a_{i} \preccurlyeq b_{i}$ then $\sum a_{i} \preccurlyeq \sum b_{i}$. Furthermore, if $\mathscr{S}$ is a partially-ordered set, then it is positive if $\varepsilon=\perp$ is the infimum or bottom for this set $\perp \preccurlyeq a$ for all $a \in S$ [23]. If $\mathscr{S}$ is positive then also if $a_{i} \preccurlyeq b_{i}$ then $\prod a_{i} \preccurlyeq \prod b_{i}[23]$.

In a semiring, the natural or canonical or difference pre-order is for all $a, b \in S$, $a \preccurlyeq b \Longleftrightarrow a \oplus c=b$ for some $c \in S$. A semiring $\mathscr{D}=\langle D, \oplus, \otimes, \perp, e\rangle$ 
is a dioid-for double monoid —or naturally_or canonically-[24] or differenceordered $[17,23,25]$ if this natural pre-order is actually a partial order.

Example 1 The following are dioids whose relationships can be gleaned from Fig. 1.

1. $\mathbb{B} \equiv \mathbb{Q} \equiv\langle\{0,1\}, \vee, \wedge, 0,1\rangle$, (Boolean algebra)

2. $\mathbb{I}_{\max , \min } \equiv\langle[0,1], \max , \min , 0,1\rangle$, (toll semiring) [26]

3. $\mathbb{N}_{\min ,+} \equiv\langle\mathbb{N} \cup\{0, \infty\}$, min, $+, \infty, 0\rangle$, (tropical semiring)

4. $\mathbb{R}_{\max ,+} \equiv\langle\mathbb{R} \cup\{-\infty\}, \max ,+,-\infty, 0\rangle$, (max-plus, schedule or morphological algebra) [27]

5. $\mathbb{R}_{\min ,+} \equiv\langle\mathbb{R} \cup\{\infty\}, \min ,+, \infty, 0\rangle$ [27], (optimization algebra)

6. $\mathbb{R}_{\max , x} \equiv\left\langle\mathbb{R}_{0}^{+} \cup\{\infty\}, \max , \times, 0,1\right\rangle$, (max-times semiring) [24]

7. $\mathbb{I}_{\max , x} \equiv\langle[0,1], \max , \times, 0,1\rangle$, (fuzzy max-times algebra) $[24]$

Note that the product of $k$ dioids is also a dioid.

A big class of dioids is that of (additively) idempotent semirings. An idempotent semiring $\mathscr{D}$ is a semiring whose additive structure $\langle D, \oplus, \varepsilon\rangle$ is an idempotent semigroup, that is, $\forall a \in D, a \oplus a=a$. Idempotent semirings are all canonically-ordered and, if commutative, they are already $\vee$-semilattices (read sup-or join-semilattice), whose operation is compatible with the canonical order $a \oplus b=a \vee b$ and selects the lowest upper bound, supremum or join [24, Chap. 1, Theorems 1 and 2].

Another class of important dioids are those with a multiplicative group structure or semifields. These are all entire, and thence information algebras.

Completeness issues in semirings deserve a lengthier treatment: a complete semiring $\mathscr{S}$ [25] is a semiring where for every (possibly infinite) family of elements $\left\{a_{i}\right\}_{i \in I} \subseteq S$ we can define an element $\sum_{i \in I} a_{i} \in S$ such that

1. if $I=\varnothing$, then $\sum_{i \in I} a_{i}=\varepsilon$,

2. if $I=\{1 \ldots n\}$, then $\sum_{i \in I} a_{i}=a_{1} \oplus \cdots \oplus a_{n}$,

3. if $b \in S$, then $b \otimes\left(\sum_{i \in I} a_{i}\right)=\sum_{i \in I} b \otimes a_{i}$ and $\left(\sum_{i \in I} a_{i}\right) \otimes b=\sum_{i \in I} a_{i} \otimes b$, and

4. if $\left\{I_{j}\right\}_{j \in J}$ is a partition of $I$, then $\sum_{i \in I} a_{i}=\sum_{j \in J}\left(\sum_{i \in I_{j}} a_{i}\right)$.

If $I$ is countable in the definitions above, then $\mathscr{S}$ is countably complete and already zerosumfree [17, Prop. 22.28].

To investigate completion issues, call an element in a semiring $a \in S$ infinite iff $a \oplus b=a$ for all $b \in S$, and strongly infinite if also $a \otimes b=a=b \otimes a$ [17]. If $\overline{\mathscr{S}}$ is a complete semiring, then it has a (necessarily unique) infinite element [17, Prop. 22.27].

A dioid $\mathscr{D}$ is complete, if it is complete as a semiring, further complete as a naturally ordered set $\langle D, \preccurlyeq\rangle$ and left $\left(\mathrm{L}_{a}\right)$ and right $\left(\mathrm{R}_{a}\right)$ multiplications are lower semicontinuous, that is, join-preserving. In such cases, the top of the dioid is the unique infinite element $\top=\top \oplus a$, for all $a \in D$, whence $a \preccurlyeq \top$.

As a partially-ordered set, a $\vee$-semilattice is complete when the lowest upper bound operates on arbitrary subsets of $S$ and likewise for complete $\wedge$-semilattices. Lattices are complete when both their $\vee$ - and $\wedge$-semilattices are complete, hence they 
have both a top and a bottom. From a well-known order-theory theorem-a complete $\checkmark$-semilattice with bottom is also a complete lattice [28, Theorem 2.31, p. 47] - it is clear that complete idempotent semirings are already complete lattices.

A fortiori, selective semifields can all be completed, as, for instance, the (initially incomplete) maxplus and minplus semifields in Example 1.

Example 2 1. The completed Minplus semifield, $\overline{\mathbb{R}}_{\min ,+}=\langle\mathbb{R} \cup\{-\infty, \infty\}$, min, $\dot{+}, \infty, 0,-\infty\rangle$

2. the completed Maxplus semifield, $\overline{\mathbb{R}}_{\max ,+}=\langle\mathbb{R} \cup\{-\infty, \infty\}$, max,, , $-\infty, 0, \infty\rangle$.

These two completions are actually inverses $\overline{\mathbb{R}}_{\min ,+}=\overline{\mathbb{R}}_{\max ,+}^{-1}$ and orderdual [29]. Indeed they are better jointly called the max-min-plus semiring $\overline{\mathbb{R}}_{\max ,+}^{\min , \dot{+}}$. We have $-\infty+\infty=-\infty$ and $-\infty \dot{+} \infty=\infty$, which solves several issues in dealing with the separately completed dioids.

Completed idempotent semifields were first recorded as blogs, bounded, latticeordered groups [30, Sect.4.1], although the name did not catch, and would be called bounded $\ell$-groups nowadays. The lattice $\mathbb{B}$ can be embedded in any bounded $\ell$ group, by restricting the carrier set to $\{\perp, \top\}$. The boolean operations would then be implemented as $\oplus$ and $\otimes$ restricted to such set.

In this context, a semimodule over a semiring, is the analogue of a module over a ring [17, 25]: a right $\mathscr{S}$-semimodule is an additive commutative monoid $\mathscr{X}=\langle X, \oplus, \varepsilon \mathscr{X}\rangle$ endowed with a right action $(x, \lambda) \mapsto x \odot \lambda$ such that $\forall \lambda, \mu \in S, x, x^{\prime} \in X$. Following the convention of dropping the symbols for the scalar action and semiring multiplication we have:

$$
\begin{aligned}
x(\lambda \mu) & =(x \lambda) \mu & & x \varepsilon=\varepsilon \mathscr{X} \\
\left(x \oplus x^{\prime}\right) \lambda & =x \lambda \oplus x^{\prime} \lambda & & x e=x
\end{aligned}
$$

The definition of a left $\mathscr{S}$-semimodule $\mathscr{Y}$ follows the same pattern with the help of a left action, $(x, \lambda) \mapsto \lambda \odot x$ and similar axioms. A $(\mathscr{R}, \mathscr{S})$-semimodule is a set $M$ endowed with left $\mathscr{R}$-semimodule and a right $\mathscr{S}$-semimodule structures, and a $(\mathscr{R}, \mathscr{S})$-bisemimodule a $(\mathscr{R}, \mathscr{S})$-semimodule such that the left and right actions commute.

For $n, p \in \mathbb{N}$, the semimodule of finite matrices $\mathscr{M}_{n \times p}(\mathscr{S})=\left\langle S^{n \times p}, \oplus, \mathscr{E}\right\rangle$ is a $\left(\mathscr{M}_{n}(\mathscr{S}), \mathscr{M}_{p}(\mathscr{S})\right)$-bisemimodule, with matrix multiplication-like left and right actions and entry-wise addition. Special cases of it are the bisemimodules of column vectors $\mathscr{M}_{p \times 1}(\mathscr{S})$ and row vectors $\mathscr{M}_{1 \times n}(\mathscr{S})$. In the following we systematically equate left (resp. right) $\mathscr{S}$-semimodules and row (resp. column) semimodules over $\mathscr{S}$. This motivates calling them (semi)vector spaces.

In a semimodule $\mathscr{X}$ over a semifield $\mathscr{K}$ one can define an element-wise inversion operation $\cdot^{-1}: X \rightarrow X, x \mapsto x^{-1}$ such that $\left(x^{-1}\right)_{i}=x_{i}^{-1}$. If the semifield is also 
a complete dioid, then the inverse semimodule is the order dual $\mathscr{X}^{-1} \cong\left\langle X, \leq^{\mathrm{d}}\right\rangle$. Hence, a complete semimodule [17] over an idempotent semifield is also a complete lattice, with join and meet operations fulfilling $v_{1} \leq v_{2} \Longleftrightarrow v_{1} \vee v_{2}=v_{2} \Longleftrightarrow$ $v_{1} \wedge v_{2}=v_{2} \cdot v_{1} \wedge v_{2}=\left(v_{1}^{-1} \vee v_{2}^{-1}\right)^{-1}$ à la Boole.

Example 3 Semimodules over $\overline{\mathbb{R}}_{\max ,+}$ have inverses over $\overline{\mathbb{R}}_{\min ,+}$ and vice versa. In particular $\left(\overline{\mathbb{R}}_{\max ,+}\right)^{-1}=\overline{\mathbb{R}}_{\min ,+}$, and dually. For $\overline{\mathbb{R}}_{\max ,+}$, it is $v_{1} \wedge v_{2}=v_{1} \dot{\oplus} v_{2}=$ $\left(v_{1}^{-1} \oplus v_{1}^{-1}\right)^{-1}=\min \left(v_{1}, v_{2}\right)$.

Note that elements of a semimodule over a semiring receive different names in different traditions: for the boolean semiring, they are called characteristic vectors; over a fuzzy algebra $L$ they are $L$-fuzzy sets; for fields or semifields they are simply vectors.

\section{Galois Connections over Semimodules and their Applications}

In this section we try to motivate why the study of Galois connections in general-and FCA in particular-should stand foremost among the applications of implications.

\subsection{Galois Connections and their Ubiquity}

A Galois connection is a pair of maps $\phi: X \rightarrow Y$ and $\psi: Y \rightarrow X$ between two ordered sets $\langle X, \leq\rangle$ and $\langle Y, \leq\rangle$ such that:

1. $x_{1} \leq x_{2} \Rightarrow \phi\left(x_{1}\right) \geq \phi\left(x_{2}\right)$

2. $y_{1} \leq y_{2} \Rightarrow \psi\left(y_{1}\right) \geq \psi\left(y_{2}\right)$

3. $x \leq \psi(\phi(x))$ and $y \leq \phi(\psi(y))$

See [11] for a summary of mathematical applications of Galois connections, and [31] for a historical review - and proof of their ubiquity. For our present interests, the composition of the maps $\gamma_{X}=\psi \circ \phi$ and $\gamma_{Y}=\phi \circ \psi$ are closure maps, that is monotone, expansive and idempotent maps. Illustrating for $\gamma_{X}$,

monotonicity: $\quad x_{1} \leq x_{2} \Rightarrow \gamma_{X}\left(x_{1}\right) \leq \gamma_{X}\left(x_{2}\right)$

expansiveness: $\quad x \leq \gamma_{X}(x)$

idempotency: $\quad \gamma_{X}\left(\gamma_{X}(x)\right)=\gamma_{X}(x)$.

A well-known order-theorem states that the set of closed elements of a closure mapcalled a closure system- $\gamma_{X}(X)=\left\{x \in X \mid \gamma_{X}(x)=x\right\}$, is a complete lattice [32, 33], a ubiquitous structure in algebra, morphological processing, rough set and fuzzy set theory, among others. 


\subsection{Activating Galois Connections and Lattices: Formal Concept Analysis}

Formal concept analysis [10] is a procedure to render lattice theory more concrete and manipulative. FCA stems from the realization that a binary relation between two sets $I \in 2^{G \times M}$ — where $G$ and $M$ are conventionally called the set of objects and attributes, respectively-defines a Galois connection between the powersets $X \equiv 2^{G}$ and $Y \equiv 2^{M}$ endowed with the inclusion order. The triple $(G, M, I)$ is called a formal context and the pair of maps that build the connection are called the polars (of the context):

$$
\begin{aligned}
& \forall A \in 2^{G}, A^{\uparrow}=\{m \in M \mid \forall g \in A, g \text { Im }\} \\
& \forall B \in 2^{M}, B^{\downarrow}=\{g \in G \mid \forall m \in B, g \text { Im }\} .
\end{aligned}
$$

The closure maps are $\gamma_{G}(A)=\left(A^{\uparrow}\right)^{\downarrow}$ and $\gamma_{M}(B)=\left(B^{\downarrow}\right)^{\uparrow}$. The closed sets of objects and attributes are called extents and intents, respectively, and their closure systems the lattice of extents and the lattice of intents.

The main theorem of formal context analysis gathers several propositions: that the lattices of extents and intents are (order-dually) isomorphic complete lattices, how the joins and meets of elements can be calculated, and, perhaps more importantly, that any complete lattice emerges in this way up to isomorphism. In this manner, any lattice can be studied by means of the lattices associated to a formal context.

The isomorphic pairs of extents and intents are called formal concepts, and model precisely the dually of extent and intent in traditional formal semantics, whence the name of the whole theory. The lattice of such pairs is called the concept lattice of the formal context $(G, M, I)$.

The existence of the Galois connection induced by a formal context gives rise to a number of information processing devices, among them the concept lattice and the bases of implications [10]. This is a flourishing field of mathematical applications with a number of conferences and special sessions dedicated to it.

\subsection{Conditional Implications and Galois Connections}

In the historical development of FCA the question soon arised whether this construction is generalizable to multi-valued relations. That is whether there is another diagram similar to Fig. 2 where characteristic vectors are substituted by vectors with entries in a semiring belonging to a semimodule. This was soon answered in the affirmative and in this construction, conditional implications are crucial.

In fact, we can prove the formal equality of Galois connections over different dioids: Given two semimodules $\mathscr{X} \equiv S^{G}$ and $\mathscr{Y} \equiv S^{M}$ over a semiring and a $S$-valued relation between them $I \in S^{G \times M}$ consider vectors $x \in S^{G}$ and $y \in S^{M}$ as $S$-sets. 

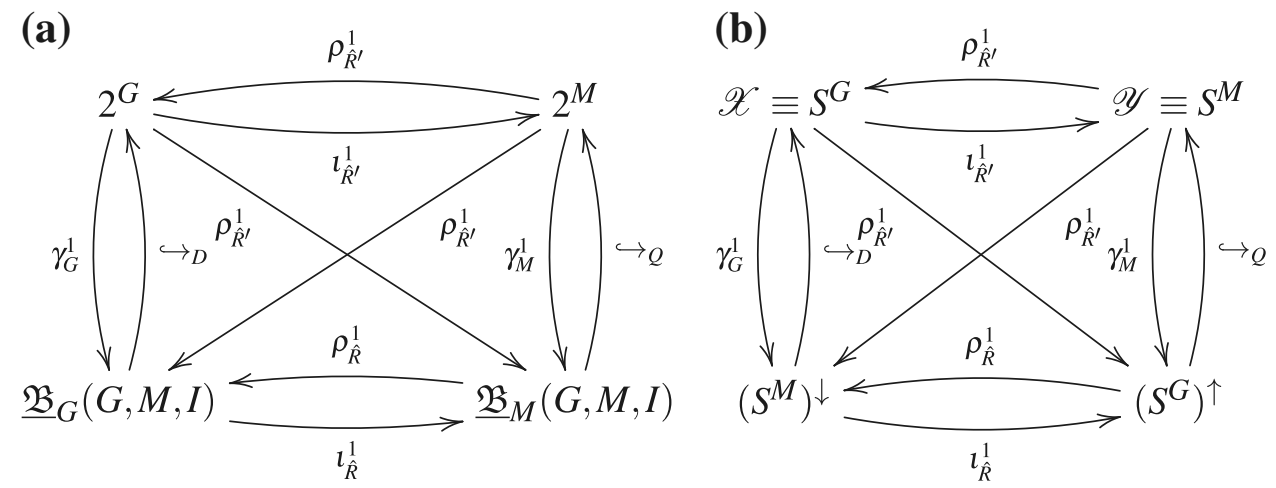

Fig. 2 The Galois connection arising in FCA or $\mathbb{B}$-FCA and an analogue over a semiring. a The Galois connection in FCA, b A Galois connection between semimodules over a semiring $S$

First, recall that when $S$ is a residuated lattice $\mathscr{L}=\langle L, \vee, \wedge, \otimes, \rightarrow, 0,1\rangle$ the polars of an L-fuzzy Galois connection between two sets of objects $G$ and attributes $M$ mediated by the fuzzy incidence $I(g, m) \in L^{G \times M}$ are [16, p.219]:

$$
\begin{aligned}
& \forall A \in L^{G}, A^{\uparrow}(m)=\bigwedge_{g \in G} A(g) \rightarrow I(g, m) \\
& \forall B \in L^{M}, B^{\downarrow}(g)=\bigwedge_{m \in M} B(m) \rightarrow I(g, m) .
\end{aligned}
$$

On idempotent semifields, however, [18] have proven that the four different types of Galois connection emerge from variations of the following construction: Given a scalar product $\langle x, y\rangle=x^{\mathrm{T}} \otimes R \otimes y$, and a $\varphi \in K$, let the $\varphi$-polars be:

$$
x^{\uparrow}=\bigvee\left\{y \in K^{M} \mid\langle x, y\rangle \leq \varphi\right\} \quad y^{\downarrow}=\bigvee\left\{x \in K^{G} \mid\langle x, y\rangle \leq \varphi\right\} .
$$

This is the definition of the residuals with respect to the scalar product which turn out to be

$$
x^{\uparrow}=\varphi /\left(x^{\mathrm{T}} \otimes R\right)=\varphi \dot{\otimes} R^{\circledast} \dot{\otimes} x^{-1} \quad y^{\downarrow}=(R \otimes y) \backslash \varphi=y^{\circledast} \dot{\otimes} R^{\circledast} \dot{\otimes} \varphi .
$$

whereby the polars are written as linear operations on a semimodule of the dual idempotent semifield. As a result, we may operate in the familiar realm of linear algebra, but maintaining our intuitions about the behavior of $K$-valued sets.

An apparent discrepancy is that the implications are not always evident, but in fact all of them follow from the residuation equation:

$$
a \otimes b \leq \varphi \Leftrightarrow a \leq \varphi / b=b \rightarrow \varphi \Leftrightarrow b \leq a \backslash \varphi=a \rightarrow \varphi .
$$


Equation (5) might look different to (4), but it is not: recall that

$$
R \dot{\otimes} y=\sum_{m \in M}^{\bullet} R(g, m) \dot{\otimes} y(m)
$$

and since the addition in idempotent semifields is the join, in the case of the inverse semifield it is actually the meet, whence we may write for $\varphi=e$,

$$
\begin{aligned}
& \forall x \in K^{G}, x^{\uparrow}(m)=\bigwedge_{g \in G} x^{-1}(g) \dot{\otimes} R^{\circledast}(g, m)=\bigwedge_{g \in G} x^{\mathrm{T}}(g) \rightarrow R^{\circledast}(g, m) \\
& \forall y \in K^{M}, y^{\downarrow}(g)=\bigwedge_{m \in M} y^{\circledast}(m) \dot{\otimes} R^{\circledast}(g, m)=\bigwedge_{m \in M} y(m) \rightarrow R^{\circledast}(g, m)
\end{aligned}
$$

where, in the last step, we have recalled that residuum is just alternate notation for the implication arrow. Note that this is formally identical to (4), mutatis mutandis.

\subsection{Applications}

Some examples of applications that can be explored under this new perspective are the visualization and analysis of contingency tables for artificial as well as human classification error assessment [34-36], analysis of Gene Expression Data [37, 38], data mining by using $\mathscr{K}-$ FCA [39] or fuzzy-FCA as in [40] for tag mining, [41] for the discovery of semantic web services, [42] for constructing user profiles from web usage logs, [43] for supporting the user in the discovery of semantic web services.

The new point of view introduced in this paper widens the scope of real-life applications of fuzzy implications since fuzzy Galois connections are at the heart of some of the generalizations of FCA. Knowledge processing, discovery, exploration and visualization as well as data mining are research areas were FCA has a niche.

\section{Conclusions}

The aim of this paper was to study the application of Galois connections in general — and formal concept analysis in particular — to conditional implication. We believe that FCA provides an extended view into fuzzy implications beyond that provided by previous works widening even more their range of real-life applications.

The main scope of this special volume of Studies in Fuzziness and Soft Computing is to pay homage to Prof. Enric Trillas, who among his many achievements in the field of fuzzy sets and systems has, without any doubt, expanded the knowledge on fuzzy implications, inspiring many of us to try to do likewise. With this paper we hope to contribute to that homage opening a new area of applications for fuzzy implications. 


\section{References}

1. Dubois, D., Prade, H.: Fuzzy sets in approximate reasoning, part 1: inference with possibility distributions. Fuzzy Sets Syst. 100(Supplement 1), 73-132 (1999)

2. Klir, G.J., Yuan, B.: Fuzzy Sets and Fuzzy Logic: Theory and Applications. Prentice-Hall Inc., Upper Saddle River (1995)

3. Trillas, E., del Campo, C., Cubillo, S.: When QM-operators are implication functions and conditional fuzzy relations. Int. J. Intell. Syst. 15(7), 647-655 (2000)

4. Yager, R.R.: On some new classes of implication operators and their role in approximate reasoning. Inf. Sci. 167(1-4), 193-216 (2004)

5. Alsina, C., Trillas, E., Valverde, L.: On some logical connectives for fuzzy sets theory. J. Math. Anal. Appl. 93, 11-26 (1983)

6. Trillas, E., Valverde, L.: On inference in fuzzy logic. In: Proceedings of the 2nd International Fuzzy Systems Association (IFSA) Congress, pp. 294-297 (1987)

7. Valverde, L., Trillas, E.: On modus ponens in fuzzy logic. In: Proceedings of 15th International Symposium on Multiple-Valued Logic, pp. 294-301 (1985)

8. Mas, M., Monserrat, M., Torrens, J., Trillas, E.: A survey on fuzzy implication functions. IEEE Trans. Fuzzy Syst. 15(6), 1107-1121 (2007)

9. Barbut, M., Monjardet, B.: Ordre et classification. Algèbre et combinatoire, tome I. Méthodes mathématiques des sciences de l'Homme. Hachette (1970)

10. Ganter, B., Wille, R.: Formal Concept Analysis: Mathematical Foundations. Springer, Berlin (1999)

11. Erné, M., Koslowski, J., Melton, A., Strecker, G.: A primer on Galois connections. In: Todd, A. (ed.) Annals of the New York Academy of Sciences, vol. 704, pp. 103-125. Madison (1993)

12. Carpineto, C., Romano, G.: Concept Data Analysis. Theory and Applications. Wiley, Chichester (2005)

13. Poelmans, J., Kuznetsov, S.O., Ignatov, D.I., Dedene, G.: Formal concept analysis in knowledge processing: a survey on models and techniques. Expert Syst. Appl. 40(16), 6601-6623 (2013)

14. Valverde-Albacete, F., Peláez-Moreno: Systems vs. methods: an analysis of the affordances of formal concept analysis for information retrieval. In: Formal Concept Analysis Meets Information Retrieval Workshop, Moscow, Russia, co-located with the 35th European Conference on Information Retrieval (ECIR 2013), pp. 113-126 (2013)

15. Burusco, A., Fuentes-González, R.: The study of the L-fuzzy concept lattice. Mathw. Soft Comput. 1(3), 209-218 (1994)

16. Bělohlávek, R.: Fuzzy Relational Systems. Foundations and Principles. IFSR International Series on Systems Science and Engineering, vol. 20. Kluwer Academic, New York (2002)

17. Golan, J.S.: Semirings and Their Applications. Kluwer Academic, Dordrecht (1999)

18. Valverde-Albacete, F.J., Peláez-Moreno, C.: Extending conceptualisation modes for generalised formal concept analysis. Inf. Sci. 181, 1888-1909 (2011)

19. Belohlavek, R., Vychodil, V.: Attribute implications in a fuzzy setting. Formal Concept Analysis, pp. 45-60. Springer, Berlin (2006)

20. Goguen, J.A.: L-fuzzy sets. J. Math. Anal. Appl 18(1), 145-174 (1967)

21. Zadeh, L.A.: Fuzzy sets. Inf. Control 8(3), 338-353 (1965)

22. Gondran, M., Minoux, M.: Dioids and semirings: links to fuzzy set and other applications. Fuzzy Sets Syst. 158, 1273-1294 (2007)

23. Golan, J.S.: Semirings and Affine Equations Over Them: Theory and Applications. Mathematics and Its Applications. Kluwer Academic Publishers, Dordrecht (2003)

24. Gondran, M., Minoux, M.: Graphs, Dioids and Semirings. New Models and Algorithms. Springer, New York (2008)

25. Golan, J.S.: Power algebras over Semirings. With Applications in Mathematics and Computer Science. Mathematics and its Applications, vol. 488. Kluwer Academic, Dordrecht (1999)

26. Cuninghame-Green, R.A., Cechlárová, K.: Residuation in fuzzy algebra and some applications. Fuzzy Sets Syst. 71(2), 227-239 (1995) 
27. Cuninghame-Green, R.A.: Minimax algebra and applications. Fuzzy Sets Syst. 41(3), 251-257 (1991)

28. Davey, B., Priestley, H.: Introduction to Lattices and Order, 2nd edn. Cambridge University Press, Cambridge (2002)

29. Moreau, J.J.: Inf-convolution, sous-additivité, convexité des fonctions numériques. J. Math. Pures et Appl. 49, 109-154 (1970)

30. Cuninghame-Green, R.: Minimax Algebra. Lecture Notes in Economics and Mathematical Systems, vol. 166. Springer, Berlin (1979)

31. Erné, M.: Adjunctions and Galois Connections: Origins, History and Development. Mathematics and Its Applications, vol. 565. Kluwer Academic, Dordrecht (2004)

32. Birkhoff, G.: Lattice Theory, 3rd edn. American Mathematical Society, Providence (1967)

33. Gratzer, G.: General Lattice Theory. Birkhauser, Berlin (2003)

34. Peláez-Moreno, C., García-Moral, A.I., Valverde-Albacete, F.J.: Analyzing phonetic confusions using formal concept analysis. J. Acoust. Soc. Am. 128(3), 1377-1390 (2010)

35. Peláez-Moreno, C., García-Moral, A.I., Valverde-Albacete, F.J.: Eliciting a hierarchical structure of human consonant perception task errors using formal concept analysis. In: Proceedings of INTERSPEECH, pp. 828-831 (2009)

36. Peláez-Moreno, C., Valverde-Albacete, F.J.: Detecting features from confusion matrices using generalized formal concept analysis. In: Hybrid Artificial Intelligence Systems, pp. 375-382. Springer, Berlin (2010)

37. González Calabozo, J., Peláez-Moreno, C., Valverde-Albacete, F.: Gene expression array exploration using $\mathscr{K}$-formal concept analysis. In: Valtchev, P., Jäschke, R. (eds.) Formal Concept Analysis. Lecture Notes in Computer Science, vol. 6628, pp. 119-134. Springer, Berlin (2011)

38. González-Calabozo, J.M., Peláez-Moreno, C., Valverde-Albacete, F.J.: WebGeneKFCA: an on-line conceptual analysis tool for genomic expression data. In: International Conference on Concept Lattices and Applications, pp. 345-350 (2012)

39. Valverde-Albacete, F.J., Peláez-Moreno, C.: Towards a generalisation of formal concept analysis for data mining purposes. In: Missaoui, R., Schmid, J. (eds.) Proceedings of the International Conference on Formal Concept Analisys. LNCS, vol. 3874, pp. 161-176. Springer (2006)

40. Chou, C.Y., Mei, H.: Analyzing tag-based Mashups with fuzzy FCA. In: IEEE International Symposium on Service-Oriented System Engineering, pp. 257-262 (2008)

41. Fenza, G., Loia, V., Senatore, S.: Concept mining of semantic web services by means of extended fuzzy formal concept analysis (FFCA). In: IEEE International Conference on Systems, Man and Cybernetics, pp. 240-245 (2008)

42. Zhou, B., Hui, S.C., Fong, A.C.M.: An effective approach for periodic web personalization. In: IEEE/WIC/ACM International Conference, pp. 284-292 (2006)

43. Fenza, G., Senatore, S.: Friendly web services selection exploiting fuzzy formal concept analysis. Soft. Comput. 14(8), 811-819 (2010) 